\title{
9 Equilibrium dynamics and local indeterminacy in a model of sustainable tourism
}

\author{
Giovanni Bella
}

\subsection{Introduction}

Tourism economics is considered a branch of standard economic theory devoted to the study of the overall class of economic events accruing from the activity of those agents called tourists. The main interest, either theoretical or empirical, concerning tourism economics lies in the useful insights it could generate in terms of policy interventions, insights that might be of help in relation to other micro- and macroeconomic actions in an overall strategy for economic development. Tourism economics is a rather new discipline, but there is explosively growing interest in it. Unfortunately, formalized theoretical bases and welldefined assumptions are still lacking in this field, making it difficult to carry out a deep investigation and exhaustive analysis of such economic facts and implications so that they can be fully documented and accurately explained.

Like every human activity, tourism is based on a relationship with the surrounding environment, influencing it and being influenced by it in a complex way (see, for example, Budowski, 1976: 27). On the other hand, as an industrial activity, tourism will have some negative effects on the environment, for tourist firms might overuse the natural resources at their disposal, making inevitable the rise of a new 'tourism curse' (e.g. Sachs and Warner, 2001: 827). Sustainable tourism is definitely a new concept in economics, as the recurrent exploitation of natural resources still constitutes the principal source of income of tourist destinations overall.

Paraphrasing the highly cited Brundtland Report's definition of sustainable development, we may therefore outline a sustainable pathway for tourism, basically saying that each human or economic activity (from the point of view both of the tourism industry and of the hosting population) must be able to meet the needs of the present generation of tourists without compromising the capabilities of future ones to satisfy their own needs (e.g. Casagrandi and Rinaldi, 2002: 13; Swarbrooke, 1999).

However, once environmental resources are taken into account, some interesting questions then suddenly arise: 'does tourism matter for economic growth?' and therefore, 'to what extent can tourism affect economic dynamics in such a way as to lead towards a long-run stable equilibrium?', or lastly, 'may eventually 
an indeterminacy problem or, alternatively, stable equilibria arise when tourism, given priority when it comes to an environmental resource, is allowed to enter the maximization problem?'.

As I have mentioned, tourism's contribution to economic development has been well documented in the literature. Nonetheless, though particular attention has been paid to the empirical exploration of the economic effects produced by tourism flows in the growth performance of different countries, there has been surprisingly little interest in studying the determinants of the same relationship from a theoretical point of view instead (e.g. Giannoni and Maupertuis, 2005; Candela and Cellini, 2006: 41).

On the one hand, for example, a recent empirical study shows that small 'tourism economies' do manifest higher levels of economic growth compared to the average for OECD countries (e.g. Brau et al., 2007; McElroy, 2006: 61). This is also confirmed by numerous other papers, even though there has not yet been a clear investigation of the interdependencies between tourism and the environment (see, for reference, Lanza and Pigliaru, 1994: 15; 2000: 77; Smeral, 2003: 77). On the other hand, Cerina (2006) has made a ground-breaking study involving deep investigation of the steady-state properties of an economy whose tourism sector is specifically based on use of a natural resource.

In the light of this still new theoretical literature, the present chapter studies the potential impact of mass tourism on the evolution of the surrounding environment in a long-lasting sustainable perspective. Indeed, its intention is to give an insight into how overuse of natural resources can be avoided without negatively affecting economic growth (see also Lozano et al., 2005).

To this end, I develop an optimal control problem where the representative agent faces a tourism-oriented economic scenario. In this framework, tourism is based on the use of existing environmental resources, but this leads to an inevitable trade-off as both positive effects (in terms of new output) and negative impacts (in terms of environmental degradation) are generated. The problem is then finally to choose the optimal number of tourists to be hosted in this economy, as well as the long-run levels of both consumption and natural resource extraction that maximize the aggregate social welfare.

As will become clear, this chapter makes use of the seminal Uzawa-Lucas model (ULM), for it has become one of the most preferred frameworks from which predictions on the growth process of two-sector economies are commonly derived in the economic literature (see, for example, Boldrin and Rustichini, 1994: 323; Ladrón-de-Guevara et al., 1997: 115; Mattana, 2004; Nishimura and Shigoka, 2006: 199). Indeed, its formal structure, accompanied by a simple mathematical characterization, offers a particularly appreciated synthesis between complexity of the topics involved and analytical tractability. I basically decide to move a step forward by substituting here human for natural capital, coupled with flows of visiting tourists, as one of the possible engines for economic growth and sustainable development.

To complete the analysis, I study the transitional dynamics of the model, and provide the whole necessary and sufficient conditions for the existence of a 
feasible steady-state equilibrium associated with positive long-run growth (e.g. Restepo-Ochoa and Vázquez, 2004: 285; Gómez, 2005). Moreover, in order to justify the hereafter assumed increase in produced output, jointly with higher levels of resource exploitation due to massive tourism arrivals in economies endowed with larger amounts of natural resources, I consider the presence of an externality factor enhancing the final sector of the economy. This choice of powerful consequences is rich: on the one hand, in fact, the natural result of the traditional growth theory, namely the stability/instability outcome in the saddlepoint sense, is not automatically achieved, and indeterminacy results when the externality from natural capital are sufficiently high. Indeed, more complicated dynamic phenomena (such as multiple equilibria) are more likely to emerge instead. I am able to prove these results analytically and to give some insights into the economic intuition behind them.

The rest of the chapter is organized as follows. In section 9.2, I derive the formal structure of the model, with particular attention to the set of preferences, the level of technology, and the link between tourism and the environment. Section 9.3 concentrates on the solution of the optimization problem and investigates deeply the stability properties of the associated steady-state solutions. A final section concludes, and a subsequent appendix provides all the necessary proofs.

\subsection{Formal structure of the model}

The model is structured as follows. First, we assume the set of preferences to be defined by a standard CES utility function in the form

$$
U=\frac{c^{1-\sigma}-1}{1-\sigma}
$$

where $c$ is the level of consumption, and $\sigma$ is the inverse of the intertemporal elasticity of substitution.

Moreover, mass tourism arrivals are allowed to enter our economy and 'feed' upon an open-access natural site whose property rights are absent or unenforced, with no individual bearing the full cost of its degradation. The result is an obvious free-riding problem, accompanied by overexploitation of the available natural resources, commonly referred to in the literature as the 'tragedy of the commons' (see Hardin, 1968: 1243).

The maximizing representative agent acting this scenario, therefore, needs to modify the two constraints on both physical and natural capital to be used in his or her long-lasting policy actions in order to account for the specific effects due to the presence of a tourism sector. To this end, we devote the rest of this section to characterizing the aforementioned constraints, particularly focusing on:

- the evolution of the available natural resources, and their link to tourism inflows;

- the level of technology. 


\subsubsection{Tourism and the environment}

In a broad sense, sustainable tourism is an industry devoted to minimizing its impact on the environment and on local culture, connected with new income and employment opportunities for the development and preservation of a site. However, tourism may have different impacts, either positive or negative, on the ecological system of a country (see, for example, Hughes, 2002: 457). As a result, it may be difficult, if not impossible, to formulate policies that allow tourism to be maintained over a long period without severely affecting the environment (e.g. Papatheodoru, 2003: 407; Hillary et al., 2001: 853). ${ }^{1}$

In this view, deriving a theoretical approach to tourism sustainability is not an easy task. To this end, we need to properly identify the link between tourism flows, $T$, and the stock of the available natural resources, $E$, for we assume here that high levels of $E$ may stimulate an increase in tourist visits, even though negatively impinging on future recreation for the environment as a whole. ${ }^{2}$

To begin with, we start our analysis by giving an explicit algebraic interpretation of the stated tourism flows. Formally, let us define tourism as a slight modification of the Schaefer harvest function:

$$
T=v E
$$

where $v \in[0,1]$ is the number (i.e. percentage) of new-coming tourists visiting (i.e. harvesting) the selected natural site, $E .^{3}$

On the other hand, without any loss of generality, evolutionary dynamics of the environmental good, $E$, is assumed here to be also influenced by tourism, $T$, and explicitly given by

$$
\dot{E}=f(E, T)=\delta E(1-T) \quad \delta \in[0,1]
$$

where $f_{T}<0$, for any increase in the number of tourists diminishes the selfreproduction capacity of the ecosystem, or rather nature's capacity to recover from tourists' resource exploitation (e.g. Smulders 1995, p. 163).

To make a whole representation of the entire dynamics described so far, we substitute (9.2) into (9.3) and let $f(\cdot)$ behave as a 3D characterization of the common Verhulst logistic function (see Verhulst, 1838: 113):

$$
\dot{E}=\delta E(1-v E) \equiv f(E, v)
$$

where $\delta$ can be interpreted as the usual parameter for the internal growth rate of a natural resource, while $v$ can be finally interpreted as a choice variable representing a measure for the carrying capacity of the place being considered. ${ }^{4}$

As a matter of fact, determining the correct tourist carrying capacity can be quite complicated. It might be worth fixing the appropriate lower bound of natural resource exploitation, below which the system incurs the risk of an inevitable qualitative deterioration (see also Bretschger and Smulders, 2007: 1). We 
can thus proxy the carrying capacity by the size of arrivals - that is, the density of tourists - that a specific destination may host per unit of land, as synthesized by $1 / v$, which is commonly referred to in the literature as a 'welcoming capacity' (e.g. Costa and Manente, 2000). ${ }^{5}$

A representation of the environmental constraint described in (9.4) is provided in Figure 9.1.

\subsubsection{Technology}

Final output, $y$, is produced by employing both physical capital, $k$, and tourism flows, $T$, according to a Cobb-Douglas production function in the form

$$
y=A k^{\alpha}(T)^{1-\alpha} E_{a}^{\gamma}
$$

where $A$ is a simple parameter of scale, $E_{a}$ represents any external effect due to the presence of a 'common pool' natural capital that no one will take account of when deciding how to allocate it in time, and $\gamma \in(-1,1)$ is an externality parameter. Basically, we are assuming that, in addition to the individual effects coming from the use of natural capital on the individual's own productivity what we may call the 'internal environmental effect' - room is left for some external effects too, denoted by $E_{a}^{\gamma}$. Specifically, we call this effect external because, even though everyone benefits from it (if it is positive), no individual will take it into account when making his or her optimal decision. ${ }^{6}$ This last assumption will, of course, become critical in the derivation of the model, and the generation of multiple equilibria with the rise of some complex indeterminacy problems.

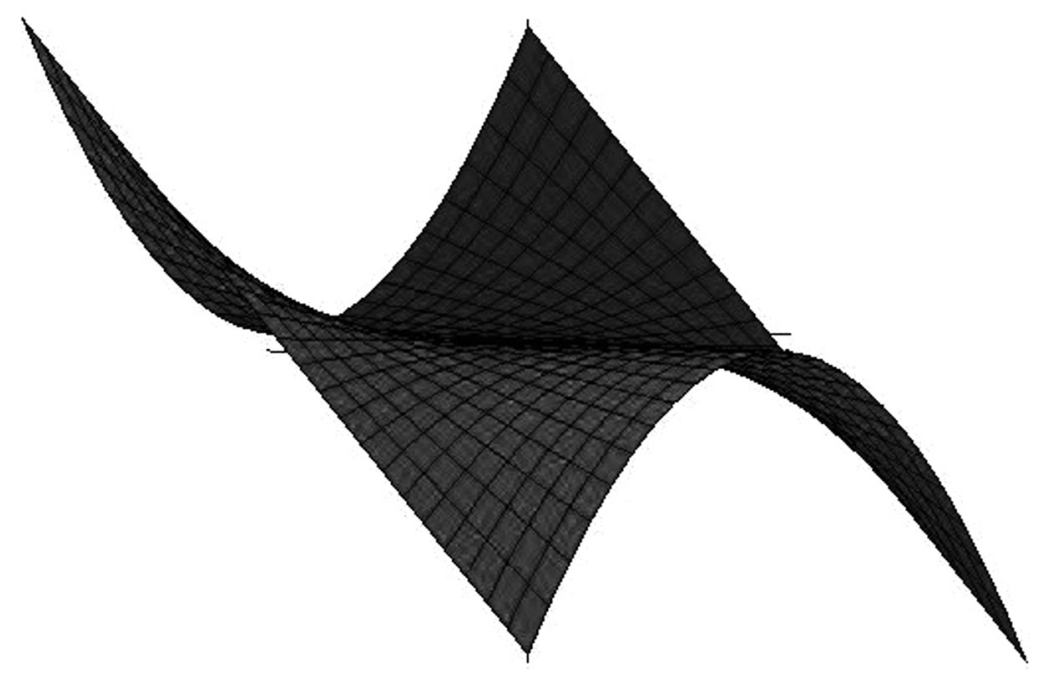

Figure 9.1 The 3D environmental constraint. 
To make the analysis simpler, we may substitute (9.2) into (9.5), and finally derive the following production function, which resembles the classic ULM specification:

$$
y=A k^{\alpha}(v E)^{1-\alpha} E_{a}^{\gamma}
$$

where final output, $y$, is expressed in terms of new visitors, $v$, as well as physical $(k)$ and natural capital $(E)$ only.

The more direct specification given in Equation 9.6 allows us, for example, to differentiate the total contribution of natural capital to production of output, $y$, in the two classical components that are commonly referred to in the literature as the use value, $E$, and the non-use value, $E_{a}$. Consequently, a deepest analysis of the external parameter, $\gamma$, may signal the impact of the non-use value on the characterization of the stability properties of our economy, as will be investigated in the rest of the chapter. ${ }^{\text {? }}$

\subsection{The optimization problem}

Formally, the representative agent maximizes the present discounted utility

$$
\max _{\mathrm{c}} \int_{0}^{\infty} \frac{c^{1-\sigma}-1}{1-\sigma} e^{-\rho t} d t
$$

subject to the following constraints on both physical and natural capital:

$$
\begin{aligned}
\dot{k} & =A k^{\alpha}(v E)^{1-\alpha} E_{a}^{\gamma}-c \\
\dot{E} & =\delta E(1-v E)
\end{aligned}
$$

given the initial conditions on each state variable:

$$
k(0)=k_{0}>0 \text { and } E(0)=E_{0}>0
$$

The current value Hamiltonian then looks like

$$
H_{C}=\frac{c^{1-\sigma}-1}{1-\sigma}+\lambda\left[A k^{\alpha}(v E)^{1-\alpha} E_{a}^{\gamma}-c\right]+\mu[\delta E(1-v E)]
$$

where $\lambda$ and $\mu$ represent the shadow prices of physical and natural capital, respectively.

The first-order condition for a maximum requires that the discounted Hamiltonian be maximized with respect to its control variables (in our case, $c$ and $v$ ):

$$
\begin{aligned}
\frac{\partial H_{C}}{\partial c} & =c^{-\sigma}-\lambda=0 \\
\frac{\partial H_{C}}{\partial v} & =\lambda(1-\alpha) A k^{\alpha} v^{-\alpha} E^{1-\alpha+\gamma}-\mu \delta E^{2}=0
\end{aligned}
$$


accompanied by the law of motion of each costate variable:

$$
\begin{aligned}
& \frac{\dot{\lambda}}{\lambda}=\rho-\alpha A k^{\alpha-1} v^{1-\alpha} E^{1-\alpha+\gamma} \\
& \frac{\dot{\mu}}{\mu}=\rho-\delta(1-v E)
\end{aligned}
$$

and the transversality condition:

$$
\lim _{t \rightarrow \infty} e^{-\rho t}\left[\lambda_{t} k_{t}+\mu_{t} E_{t}\right]=0
$$

that jointly constitute the canonical system. ${ }^{8}$

Proposition 1 The maximum principle associated with the decentralized optimization problem implies the following four-dimensional system of first-order differential equations:

$$
\begin{aligned}
& \xi_{k}=\frac{\dot{k}}{k}=A k^{\alpha-1} v^{1-\alpha} E^{1-\alpha+\gamma}-\frac{c}{k} \\
& \xi_{E}=\frac{\dot{E}}{E}=\delta(1-v E) \\
& \xi_{c}=\frac{\dot{c}}{c}=-\frac{\rho}{\sigma}+\frac{\alpha}{\sigma} A k^{\alpha-1} v^{1-\alpha} E^{1-\alpha+\gamma} \\
& \xi_{v}=\frac{\dot{v}}{v}=(\gamma-\alpha) \frac{\delta}{\alpha}(1-v E)-\frac{c}{k}
\end{aligned}
$$

Proof: see the appendix.

Lemma 1 The system S1 implies also the following reduced version:

$$
\begin{aligned}
& \dot{x}=-\frac{\rho}{\sigma} x+\left(\frac{\alpha-\sigma}{\sigma}\right) m x+x^{2} \\
& \dot{q}=\frac{\gamma \delta}{\alpha}(1-q) q-x q \\
& \dot{m}=(\alpha-1) m^{2}+\frac{\gamma \delta}{\alpha}(1-q) m
\end{aligned}
$$

by means of the convenient variable substitutions: $x=c / k, q=v E$, and $m=y / k$. 


\section{G. Bella}

Lemma 2 The steady state is a triplet $\left(x^{*}, q^{*}, m^{*}\right)$ which solves the reduced system S2:

$$
\begin{aligned}
& m^{*}=\frac{\rho}{\alpha(1-\sigma)} \\
& x^{*}=\frac{\rho(1-\alpha)}{\alpha(1-\sigma)} \\
& q^{*}=1-\frac{\rho(1-\alpha)}{\gamma \delta(1-\sigma)}
\end{aligned}
$$

given $0<\sigma<1$.

Moving a step backwards, it is worth noting that $q^{*}$ simply represents the optimal percentage of tourists allowed to enter the natural site $\left(T^{*}=q^{*}\right)$, re-expressed in terms of parameters only, and whose magnitude depends on the sign of the externality parameter, $\gamma\left(\partial T^{*} / \partial \gamma>0\right)$, as clearly depicted in Figure 9.2.

Remark 1 A negative externality on natural capital $(\gamma<0)$ drives a positive $q^{*}$ off the unit threshold $\left(q^{*}>1\right)$. Unbounded mass-tourism flows are thus booming in our economy.

Remark 2 On the contrary, a positive externality - or rather $\gamma>\frac{\rho(1-\alpha)}{\delta(1-\sigma)}-$

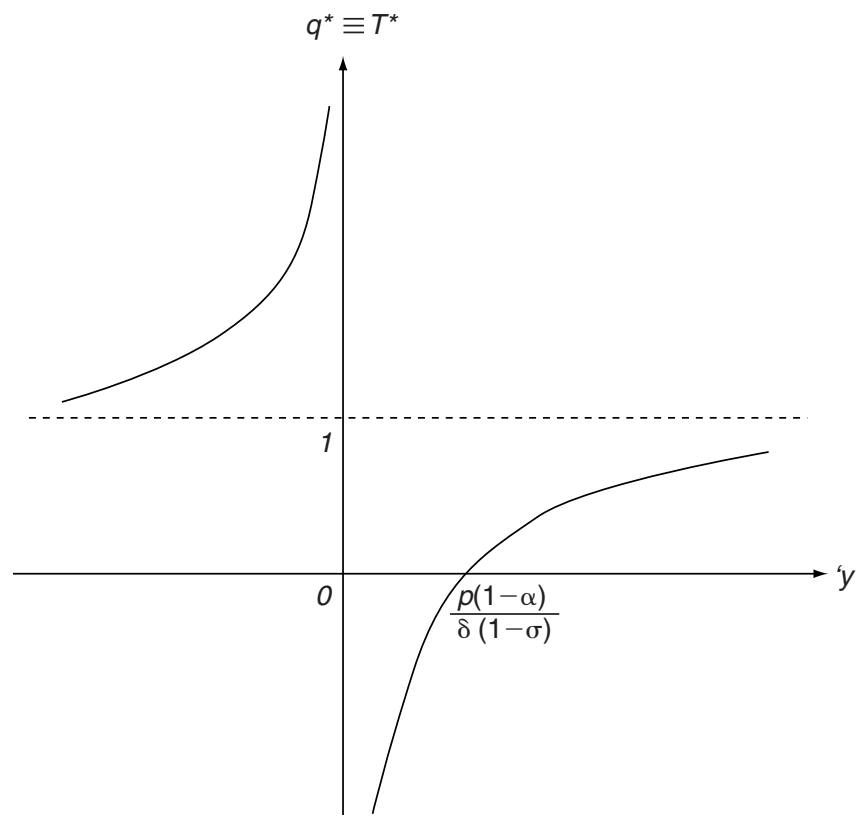

Figure 9.2 Evolution of $q^{*}=q^{*}(\gamma)$. 
always leaves a positive $q^{*}$ within the unit threshold $\left(q^{*}<1\right)$. Consequently, tourism arrivals are bounded above. Remark 3 Lastly, externality values lying in the interval $0<\gamma<\frac{\rho(1-\alpha)}{\delta(1-\sigma)}$ imply a
negative flow of tourist arrivals $\left(T^{*}<0\right)$.

The externality $\gamma$ therefore plays a crucial role in the characterization of an optimal solution to our maximization problem, and does definitely matter in the process of a growth-leading tourism. It can be highly interesting to carefully analyse the path to be followed by our economy to achieve a long-run 'sustainable' stability. The next subsection is devoted to this end.

\subsubsection{Stability properties of the steady state}

Which path will this economy follow while converging to the steady state? Is our system stable or unstable? And if it is stable, do solutions describe uniqueness or multiplicity of equilibria, or might we face indeterminacy problems? To answer these questions, we ought to investigate the local stability properties of the solution found in the previous section and describe the reasons why an indeterminate equilibrium could possibly arise. Our scope is then to determine more precisely what kind of external effect is operating in the possible generation of multiple equilibria. To this end, we analyse the Jacobian matrix $\left(J^{*}\right)$ of the reduced system $S 2$, and check for the sign of the associated eigenvalues.

Proposition 2 Let assume the following restrictions on parameters: $\delta>0,0<\alpha<$ $1,0<\sigma<1$; then there is always a continuum of equilibria if, and only if, $\gamma>\frac{\rho(1-\alpha)}{\delta(1-\sigma)}$; that is, $J^{*}$ has one positive eigenvalue and two eigenvalues with negative real parts.

Proof: Provided that $t r J^{*}>0, B J^{*}<0$, and $\operatorname{DetJ}^{*}>0$, we can thus check for local stability of the system around the steady state by means of the neat RouthHurwitz theorem. ${ }^{9}$

Proposition 3 On the other hand, if $\gamma<0$ then the equilibrium is locally unique, thus $J^{*}$ has one negative eigenvalue and two eigenvalues with positive real parts.

Proof: Repeating the same argument, we derive the sequence $t r J^{*}>0, B J^{*}=$ ?, and $\operatorname{Det}^{*}<0$, which implies now having two changes of sign, and a subsequent unique equilibrium. In this case, mass tourism is overshooting, the visited place is becoming too crowded, while the quality of the environment is dramatically decreasing.

Proposition 4 On the contrary, if $0<\gamma<\frac{\rho(1-\alpha)}{\delta(1-\sigma)}$ then the equilibrium is locally unique, thus $J^{*}$ has one negative eigenvalue and two eigenvalues with positive real parts. 
Proof: In this case, the sequence $t r J^{*}>0, B J^{*}=$ ?, and $D e t J^{*}<0$ resembles the previous outcome, although here the flow of tourists becomes negative $\left(q^{*}=T^{*}\right.$ $<0$ ), with a decreasing percentage of newcoming visitors entering our natural site, whose regenerative capacities are consequently preserved.

We can finally synthesize the results of our analysis by means of Table 9.1, where the size of the externality parameter, $\gamma$, the flow of tourists, $T$, and the evolution of the environment, $E$, are jointly represented:

Basically, the presence of multiple equilibria has been used in the literature to explain the diversity of growth rates and income differences across different countries (e.g. Xie, 1994: 97; Benhabib and Perli, 1994: 113). It is so worth noting in this chapter that the existence of a positive externality accruing to the final output is not a necessary condition for determinacy of the equilibria. ${ }^{10}$ Instead, depending on the magnitude of the externality parameter, either multiple or unique equilibria may consequently arise.

More specifically, we have found that, given the presence of a positive externality, the only way to allow for an increasing percentage of tourist arrivals within the carrying capacity of the environment needs a specific constraint on the externality parameter either, $\gamma>\frac{\rho(1-\alpha)}{\delta(1-\sigma)}$. In this case, in fact, the inward flow of tourists is upper-bounded, and no indiscriminate entries are allowed. Moreover, a positive evolution of the environment is achieved, leaving intact its regenerative capacities for the generations coming afterwards. Indeed, a positive sustainable growth rate of long-run consumption, as well as a positive inward flow of tourists with no overexploitation of the natural resources at disposal, do hopefully occur.

Nevertheless, this positive outcome is accompanied by some unexpected consequences, for indeterminacy problems and multiple equilibria do unfortunately arise.

To conclude, as is commonly assumed in the related literature, the implications of indeterminacy problems in this chapter can be synthesized as follows: two identically endowed economies with identical initial conditions may consume, and invest in the production of, natural and physical capital at completely different rates. Only in the long run will those economies converge to the same growth rate, but not to the same level of output and natural and physical capital. It is therefore possible to consider other cultural, historical or non-

Table 9.1 Results of the equilibrium analysis

\begin{tabular}{lll}
\hline$\gamma<0$ & $T^{*}=\nu E>1$ & $E_{t}=E_{0} e^{-\varphi t}$ \\
$0<\gamma<\frac{\rho(1-\alpha)}{\delta(1-\sigma)}$ & $\begin{array}{l}T^{*}=\nu E<0 \\
\text { (unique equilibrium) }\end{array}$ & $E_{t}=E_{0} e^{\varphi t}$ \\
$\gamma>\frac{\rho(1-\alpha)}{\delta(1-\sigma)}$ & $\begin{array}{l}T^{*}=\nu E<1 \\
\text { (multiple equilibrium) }\end{array}$ & $E_{t}=E_{0} e^{\varphi t}$ \\
\hline
\end{tabular}


economic factors as the means for equilibria to differ on the transition path to be followed. Indeed, we refer here to local indeterminacy, and the coexistence of multiple balanced growth paths, as the device by which to theoretically reinterpret the possibility for different regions, identically endowed in terms of existing natural resources, to exhibit uneven economic development when a tourism sector is allowed to use the visited place in, hopefully, a sustainable way.

The positive implication of this chapter can be the following: given the different allocation of natural resources across countries, and assuming that multiple equilibria may exist, it is no wonder that a clear convergence among the world's economies is not observed. In the management of their natural resources, we may notice instead that, meanwhile, some countries have lagged permanently behind as a result of short-sighted policies, while others have experienced higher growth rates as a result of adopting more sustainable behaviour. It might be that a historically stagnant region continues to be so, while other regions, perhaps historically more active, may continue to flourish, even though they are the same in all other respects. History matters, then, and the management of the natural resources, perhaps directed through tourism policies, may thus act as a selection device among these different equilibria.

Further, it can be worth noting that a positive relationship does exist between the externality parameter, $\gamma$, and the inverse of the intertemporal elasticity of substitution, $\sigma$ - that is, $\partial \gamma / \partial \sigma>0 .{ }^{11}$ But we commonly know that the higher is $\sigma$, the less willing are supposed households to accept a deviation from a uniform pattern of consumption over time (see Barro and Sala-i-Martin, 2004). Therefore, as considered before, if we let $\gamma$ represent the impact of non-use value on output production, we can finally state that a rise in $\sigma$ must necessarily be accompanied by an increase in the bequest value of the natural resource we are dealing with, if we want an optimal solution that can be maintained over time or, in other words, that the value assigned to the natural capital will rise as the need for a constant consumption increases, if we want all generations behave in a sustainable way.

\subsection{Concluding remarks}

The literature on tourism unfortunately lacks a sophisticated theoretical foundation, even though new work has been recently issued in this field to bridge the gap. To shed some light in this field, I have presented a model to answer the question of whether countries with similar tourist industries may exhibit very different growth experiences, arguing that a crucial aspect for the occurrence of both indeterminacy and cyclical adjustment towards the steady state might be the presence of an externality associated with natural capital in the production of final output.

Many works in macroeconomics have focused on models with multiple, or indeterminate, equilibria (see, among others, Benhabib and Perli, 1994: 113; Boldrin and Rustichini, 1994: 323; Matsuyama, 1991: 587; Boldrin et al., 2001: 97), though never in the field of tourism economics, to the best of my knowledge. In such macroeconomic models, the resulting possibility of a 
continuum of equilibria, or indeterminacy of equilibria, arises because of market imperfections that may come from increasing returns to scale in production, often driven by external effects. It is therefore the nature of such non-competitive markets that allows for a multiplicity of equilibria.

I follow the same approach in this chapter and provide a model to better determine the properties of such external effects in generating multiple equilibria, whenever the presence of a tourism sector is assumed to influence the fundamentals of our economy. Conclusions to the analysis confirm that such externality matters in the transition towards a long-run sustainable equilibrium, but only after a certain thresholdhas been passed, thus leaving space for other, more complicated dynamic phenomena.

\section{Appendix}

Given the following current value Hamiltonian:

$$
H_{C}=\frac{c^{1-\sigma}-1}{1-\sigma}+\lambda\left[A k^{\alpha}(v E)^{1-\alpha} E_{a}^{\gamma}-c\right]+\mu[\delta E(1-v E)]
$$

where $\lambda$ and $\mu$ represent the shadow prices of physical and natural capital, respectively, the first-order condition for a maximum requires that the discounted Hamiltonian be maximized with respect to its control variables - that is:

$$
\begin{aligned}
& \frac{\partial H_{C}}{\partial c}=c^{-\sigma}-\lambda=0 \\
& \frac{\partial H_{C}}{\partial v}=\lambda(1-\alpha) A k^{\alpha} v^{-\alpha} E^{1-\alpha+\gamma}-\mu \delta E^{2}=0
\end{aligned}
$$

accompanied by the law of motion of each costate variable

$$
\begin{aligned}
& \frac{\dot{\lambda}}{\lambda}=\rho-\alpha A k^{\alpha-1} v^{1-\alpha} E^{1-\alpha+\gamma} \\
& \frac{\dot{\mu}}{\mu}=\rho-\delta(1-v E)
\end{aligned}
$$

Moreover, since either Arrow's or Mangasarian's second-order conditions both drive to a complex calculation, we take advantage of a more general sufficient condition, following Asada et al. (1998), according to which the optimal control problem is maximized whenever the Hamiltonian function is jointly concave in the control variables, as we can easily verify in our problem, for the minors of the Hessian matrix associated to $H_{C}$

$$
\mathbf{H}=\left[\begin{array}{ll}
\frac{\partial^{2} H_{C}}{\partial c^{2}} & \frac{\partial^{2} H_{C}}{\partial c \partial v} \\
\frac{\partial^{2} H_{C}}{\partial \nu \partial c} & \frac{\partial^{2} H_{C}}{\partial v^{2}}
\end{array}\right]
$$


do exhibit the following sequence $\mathbf{H}_{1}<0$, and $\mathbf{H}_{2}>0$, given $\frac{\partial^{2} H_{c}}{\partial c^{2}}=-\sigma c^{-\sigma-1}$, $\frac{\partial^{2} H_{c}}{\partial v^{2}}=-\lambda \alpha(1-\alpha) A k^{\alpha} v^{-\alpha} E^{1-\alpha+\gamma}$, and $\frac{\partial^{2} H_{c}}{\partial c \partial v}=\frac{\partial^{2} H_{c}}{\partial v \partial c}=0$.

To add more, given the constraints on both physical and natural capital:

$$
\begin{aligned}
\dot{k} & =A k^{\alpha}(v E)^{1-\alpha} E_{a}^{\gamma}-c \\
\dot{E} & =\delta E(1-v E)
\end{aligned}
$$

and rearranging equations (9.A1)-(9.A4) in terms of growth rates, with a little bit of mathematical manipulation we can derive the four-dimensional system of first-order differential equations, $S 1$ :

$$
\begin{aligned}
& \xi_{k}=\frac{\dot{k}}{k}=A k^{\alpha-1} v^{1-\alpha} E^{1-\alpha+\gamma}-\frac{c}{k} \\
& \xi_{E}=\frac{\dot{E}}{E}=\delta(1-v E) \\
& \xi_{c}=\frac{\dot{c}}{c}=-\frac{\rho}{\sigma}+\frac{\alpha}{\sigma} A k^{\alpha-1} v^{1-\alpha} E^{1-\alpha+\gamma} \\
& \xi_{v}=\frac{\dot{v}}{v}=(\gamma-\alpha) \frac{\delta}{\alpha}(1-v E)-\frac{c}{k}
\end{aligned}
$$

or rather the more tractable reduced system, $S 2$ :

$$
\begin{aligned}
& \dot{x}=-\frac{\rho}{\sigma} x+\left(\frac{\alpha-\sigma}{\sigma}\right) m x+x^{2} \\
& \dot{q}=\frac{\gamma \delta}{\alpha}(1-q) q-x q \\
& \dot{m}=(\alpha-1) m^{2}+\frac{\gamma \delta}{\alpha}(1-q) m
\end{aligned}
$$

by means of the convenient variable substitution: $x=c / k, q=v E$, and $m=y / k$, with the associated optimal steady-state values:

$$
\begin{aligned}
m^{*} & =\frac{\rho}{\alpha(1-\sigma)} \\
x^{*} & =\frac{\rho(1-\alpha)}{\alpha(1-\sigma)} \\
q^{*} & =1-\frac{\rho(1-\alpha)}{\gamma \delta(1-\sigma)}
\end{aligned}
$$

whose positiveness requires $0<\sigma<1$. 
The Jacobian matrix of the reduced system $S 2$ is then

$$
J=\left[\begin{array}{lll}
J_{11} & J_{12} & J_{13} \\
J_{21} & J_{22} & J_{23} \\
J_{31} & J_{32} & J_{33}
\end{array}\right]
$$

where:

$$
\begin{aligned}
& J_{11}=x ; J_{12}=0 ; J_{13}=\left(\frac{\alpha-\sigma}{\sigma}\right) x ; \\
& J_{21}=-q ; J_{22}=-\frac{\gamma \delta}{\alpha} q ; J_{23}=0 ; \\
& J_{31}=0 ; J_{32}=-\frac{\gamma \delta}{\alpha} m ; J_{33}=(\alpha-1) m .
\end{aligned}
$$

Hence, the Jacobian evaluated at the steady state finally becomes

$$
J_{\left(x^{*}, q^{*}, m^{*}\right)}=\left[\begin{array}{ccc}
\frac{\rho(1-\alpha)}{\alpha(1-\sigma)} & 0 & \frac{\rho(1-\alpha)(\alpha-\sigma)}{\alpha \sigma(1-\sigma)} \\
1-\frac{\rho(1-\alpha)}{\gamma \delta(1-\sigma)} & \frac{\rho(1-\alpha)}{\alpha(1-\sigma)}-\frac{\gamma \delta}{\alpha} & 0 \\
0 & -\frac{\rho \gamma \delta}{\alpha^{2}(1-\sigma)} & \frac{\rho(\alpha-1)}{\alpha(1-\sigma)}
\end{array}\right]
$$

and therefore

$$
\begin{aligned}
& \operatorname{tr} J=\frac{\rho(1-\alpha)}{\alpha(1-\sigma)}-\frac{\gamma \delta}{\alpha}<0 \\
& \text { Det } J=\frac{\gamma \delta(1-\sigma)}{\sigma} m x q>0 \\
& B J=-\frac{\rho^{2}(1-\alpha)^{2}}{\alpha^{2}(1-\sigma)^{2}}<0 \\
& -B J+\frac{\text { Det } J}{t r J}=\frac{1}{t r J} \frac{\rho^{2}(1-\alpha)}{\sigma \alpha^{3}(1-\sigma)^{3}}[\rho(1-\alpha)-\gamma \delta(1-\sigma)](\sigma-\alpha)
\end{aligned}
$$

whose values may be useful to check for stability of the system by means of the aforementioned Routh-Hurwitz criterion.

\section{Notes}

1 An extensive interpretation of the well-known Butler theory provides us with three different scenarios that may eventually occur. In an unexplored area, the tourists are initially few in number and their number grows very slowly (exploration). Following this discovery period, there is a phase of rapid growth, in general accompanied by concomitant capital development (and resource harvesting), and finally a stagnation phase with environmental degradation (see Butler, 1980: 5).

2 To avoid confusion in the terminology used in this chapter, we will alternatively consider the terms 'natural capital', 'stock of natural resources' or 'environmental quality' as possible synonymns to identify the same environmental variable $E$. 
3 The so-called Schaefer harvesting production function basically states that $H=\alpha S L H$, where $H$ is the harvest of the natural stock, $S$, supplied to production by the $L H$ labour force used in resource harvesting, and $\alpha$ is a positive constant parameter, commonly referred to as the 'harvestability' coefficient, that hereafter we set to unity for the sake of simplicity (see Schaefer, 1957: 669).

4 The famous Verhulst logistic equation describing the evolution of an $x$-population commonly takes the form

$$
\dot{x}=r x\left(1-\frac{x}{K}\right)
$$

where $r$ is called the intrinsic growth rate, and $K$ is referred to as the carrying capacity, or the saturation level, of the natural site being considered. In our case, we simply assume that $r=\delta$, and $v=1 / K$.

5 Some authors, such as Pittel (2003), either leave $v$ out of the analysis or assume $f(\cdot)$ to be strictly concave in $E(f E E<0)$ and of an inverted $U$-shape, with a maximum at a certain point called $E^{*}$ ( $f E>0$ for $E<E^{*}$ and $f E<0$ for $E>E^{*}$ ). This type of regeneration function was originally adopted to describe the population dynamics of fish stocks and other renewable resources, and later applied to the analysis of aggregate stocks of natural resources too (Smith, 1968: 409). A broad justification for this application follows from physics and is provided by Bovenberg and Smulders (1996). Conversely, others, such as Musu (1995), propose a linear representation of the regeneration function in (9.4), even though Rosendahl (1996) argues that it could be reasonably useful for those analyses where only the positively sloped arm of the hump-shaped regeneration function is of interest. Following the same argument, Aghion and Howitt (1998) provide an approximation for the development of nature along the negatively sloped arm of the hump-shaped function.

6 Various interpretations of the externality parameter, $\gamma$, can be given. Let us think, for example, of the positive spillover effect coming to the Sardinian archipelago of $\mathrm{La}$ Maddalena thanks to the presence of the beautiful, though undisposable, site of the famous 'pink beach'. On the contrary, consider the negative impact accruing to a natural site as a result of a severe climate change (see, for example, Berritella et al., 2006: 913; and Hamilton et al., 2005: 253).

7 The use value is derived from the direct utilization of the resource being considered, while the non-use value is generally referred to as the existence, or bequest, value that people derive from the intergenerational availability of the same resource (see, for example, Turner et al., 1994).

8 A sufficient condition for concavity of the optimization problem is fully provided in the appendix to this chapter.

9 Given the characteristic equation associated with system $S 2$,

$$
-\kappa^{3}+\operatorname{tr} J^{*} \kappa^{2}-B J^{*} \kappa+\operatorname{Det}^{*}=0
$$

where $\kappa$ is the eigenvalue of the system, the Routh-Hurwitz theorem states that the number of roots of the characteristic polynomial with positive real parts is equal to the number of variations of sign in the scheme

$$
-1 t r J^{*}-B J^{*}+\frac{\operatorname{DetJ}^{*}}{\operatorname{trJ} J^{*}} \operatorname{Det} J^{*}
$$

10 The plausibility of indeterminacy problems due to the presence of externalities has been investigated in the empirical literature (see, for example, Caballero and Lyons, 1992: 209; Domowitz et al., 1988: 55; Harrison, 2003: 963).

11 The result comes by implicitly differentiating the function expressing the optimal value of tourist arrivals, $q^{*}$, previously derived in Lemma 2. 


\section{References}

Aghion, P. and Howitt, P. (eds) (1998) Endogenous Growth Theory, 2nd ed., Cambridge, MA: MIT Press.

Asada, T., Semmler, W. and Novak, A. J. (1998) 'Endogenous growth and the balanced growth equilibrium', Research in Economics, 52: 189-212.

Barro, R. J. and Sala-i-Martin, X. (eds) (2004) Economic Growth, Cambridge, MA: MIT Press.

Benhabib, J. and Perli, R. (1994) 'Uniqueness and indeterminacy: on the dynamics of endogenous growth', Journal of Economic Theory, 63: 113-142.

Berritella, M., Bigano, A., Roson, R. and Tol, R. S. J. (2006) 'A general equilibrium analysis of climate change impacts on tourism', Tourism Management, 27 (5): 913-924.

Boldrin, M. and Rustichini, A. (1994) 'Growth and indeterminacy in dynamic models with externalities', Econometrica, 62: 323-342.

Boldrin, M., Nishimura, K., Shigoka, T. and Yano, M. (2001) 'Chaotic equilibrium dynamics in endogenous growth models', Journal of Economic Theory, 96 (1-2): 97-132.

Bovenberg, A. L. and Smulders, S. (1996) 'Transitional impacts of environmental policy in an endogenous growth model', International Economic Review, 37: 861-893.

Brau, R., Lanza, A. and Pigliaru, F. (2007) 'How fast are small tourism countries growing? Evidence for the data 1980-2003', Tourism Economics, 13 (4): 603-614.

Bretschger, L. and Smulders, S. (2007) 'Sustainable resource use and economic dynamics', Environmental and Resource Economics, 36 (1): 1-13.

Budowski, G. (1976) 'Tourism and environmental conversation: conflict, coexistence or symbiosis?', Environmental Conversation, 3 (1): 27-31.

Butler, R. (1980) 'The concept of a tourist area cycle of evolution', Canadian Geographer, 24: 5-12.

Caballero, R. J. and Lyons, R. K. (1992) 'External effects in US productivity', Journal of Monetary Economics, 29: 209-226.

Candela, G. and Cellini, R. (2006) 'Investment in tourism market: a dynamic model of differentiated oligopoly', Environmental and Resource Economics, 35: 41-58.

Casagrandi, R. and Rinaldi, S. (2002) 'A theoretical approach to tourism sustainability', Conservation Ecology, 6 (1): 13-27.

Cerina, F. (2007) 'Tourism specialization and environmental sustainability in a dynamic economy', Tourism Economics, 13 (4): 553-582.

Costa, P. and Manente, M. (eds) (2000) Economia del turismo, Milan: Touring University Press.

Domowitz, I. R., Hubbard, G. and Peterson, B. C. (1988) 'Market structure and cyclical fluctuations in US manufacturing', Review of Economics and Statistics, 70: 55-66.

Giannoni, S. and Maupertuis, M. A. (2005) 'Environmental quality and long run tourism development a cyclical perspective for small island tourist economies', FEEM Working Paper, 145.05.

Gómez, M. A. (2005) 'Transitional dynamics in an endogenous growth model with physical capital, human capital and R\&D', Studies in Nonlinear Dynamics and Econometrics, 9 (1).

Hamilton, J. M., Maddison, D. J. and Tol, R. S. J. (2005) 'Climate change and international tourism: a simulation study', Global Environmental Change, 15: 253-266.

Hardin, G. (1968) 'The tragedy of the commons', Science, 162: 1243-1248.

Harrison, S. G. (2003) 'Returns to scale and externalities in the consumption and investment sectors', Review of Economic Dynamics, 6 (4): 963-976. 
Hillary, M., Nancarrow, B., Griffin, G. and Syme, G. (2001) 'Tourist perception of environmental impact', Annals of Tourism Research, 28 (4): 853-867.

Hughes, G. (2002) 'Environmental indicators', Annals of Tourism Research, 29: 457-477.

Ladrón-de-Guevara, A., Ortigueira, S. and Santos, M. S. (1997) 'Equilibrium dynamics in two-sector models of endogenous growth', Journal of Economic Dynamics and Control, 21: 115-143.

Lanza, A. and Pigliaru, F. (1994) 'The tourism sector in the open economy', Rivista Internazionale di Scienze Economiche e Commerciali, 41: 15-28.

Lanza, A. and Pigliaru, F. (2000) 'Tourism and economic growth: does country's size matter?', Rivista Internazionale di Scienze Economiche e Commerciali, 47: 77-85.

Lozano, J., Gómez, C. and Rey-Maquieira, J. (2005) 'An analysis of the evolution of tourism destinations from the point of view of the economic growth theory', FEEM Working Paper, 146.2005.

McElroy, J. L. (2006) 'Small island tourist economies across the life cycle', Asia Pacific Viewpoint, 47 (1): 61-77.

Matsuyama, K. (1991) 'Increasing returns, industrialization, and indeterminacy of equilibrium', Quarterly Journal of Economics, 106: 587-597.

Mattana, P. (ed.) (2004) The Uzawa-Lucas Endogenous Growth Model, Aldershot, UK: Ashgate.

Musu, I. (1995) 'Transitional dynamics to optimal sustainable growth', FEEM Working Paper, 50.95.

Nishimura, K. and Shigoka, T. (2006) 'Sunspots and Hopf bifurcations in continuous time endogenous growth models', International Journal of Economic Theory, 2 (3-4): 199-216.

Papatheodoru, A. (2003) 'Modelling tourism development: a synthetic approach', Tourism Economics, 9: 407-430.

Pittel, K. (ed.) (2003) Sustainability and Endogenous Growth, Cheltenham, UK: Edward Elgar.

Restrepo-Ochoa, S. I. and Vázquez, J. (2004) 'Cyclical features of the Uzawa-Lucas endogenous growth model', Economic Modelling, 21: 285-322.

Rosendahl, K. E. (1996) 'Does improved environmental policy enhance economic growth?', Environmental and Resource Economics, 9: 341-364.

Sachs, J. D. and Warner, A. M. (2001) 'Natural resources and economic development: the curse of natural resources', European Economic Review, 45: 827-838.

Schaefer, M. B. (1957) 'Some considerations of population dynamics and economics in relation to the management of marine fishes', Journal of the Fisheries Research Board of Canada, 14: 669-681.

Smeral, E. (2003) 'A structural view of tourism growth', Tourism Economics, 9: 77-93.

Smith, V. L. (1968) 'Economics of production from natural resources', American Economic Review, 58: 409-431.

Smulders, S. (1995) 'Environmental policy and sustainable economic growth: an endogenous growth perspective', De Economist, 143: 163-195.

Swarbrooke, J. (1999) Sustainable Tourism Management, Wallingford, UK: CABI.

Turner, K. R., Pearce, D. and Bateman, I. (1994) Environmental Economics: An Elementary Introduction, Baltimore: Johns Hopkins University Press.

Verhulst, P. F. (1838) 'Notice sur la loi que la population suit dans son accroissement', Correspondance Mathématique et Physique, 10: 113-121.

Xie, D. (1994) 'Divergence in economic performance: transitional dynamics with multiple equilibria', Journal of Economic Theory, 63: 97-112. 